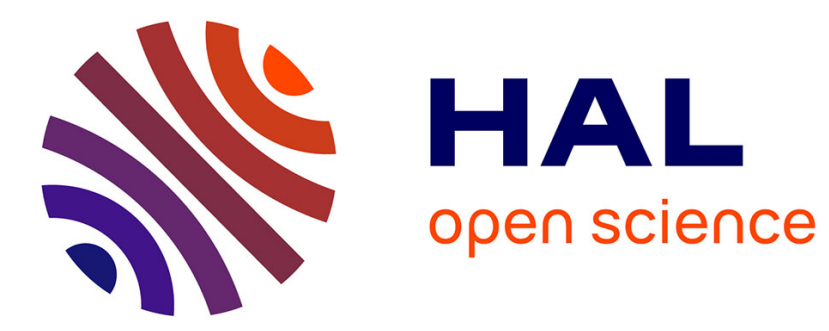

\title{
Pour une approche enactive de la parole dans les langues
}

Didier Bottineau

\section{To cite this version:}

Didier Bottineau. Pour une approche enactive de la parole dans les langues. Langages, 2013,192 (4), 10.3917/lang.192.0011 . hal-01812588

\section{HAL Id: hal-01812588 \\ https://hal.science/hal-01812588}

Submitted on 11 Jun 2018

HAL is a multi-disciplinary open access archive for the deposit and dissemination of scientific research documents, whether they are published or not. The documents may come from teaching and research institutions in France or abroad, or from public or private research centers.
L'archive ouverte pluridisciplinaire HAL, est destinée au dépôt et à la diffusion de documents scientifiques de niveau recherche, publiés ou non, émanant des établissements d'enseignement et de recherche français ou étrangers, des laboratoires publics ou privés. 


\title{
Didier Bottineau (CNRS, UMR 7114 Modyco \& Université Paris Ouest) :
}

\author{
Pour une approche enactive de la parole dans les langues
}

\section{Introduction}

Le cliché est fondateur: le mot langue désigne à la fois l'organe le plus utilisé comme articulateur actif dans la production de la parole et l'ensemble formalisé de ressources qu'un mode de parole donné utilise pour se mettre en œuvre (le lexique, la grammaire). Dans son acception technique, le mot langue en est venu à désigner tout ce que la parole n'est pas, à savoir (i) la formalisation de formes lexicales, morphologiques et syntaxiques réifiées, constituées en objets d'étude envisageables hors de leur exécution motrice et sensorielle, et (ii) la formalisation de processus génératifs (ceux des structures syntaxiques pour la grammaire générative, ceux des actes de représentation pour la psychomécanique du langage, envisageables, ici encore, indépendemment ou en amont des actes « linguaux ». L'affirmation va peut-être sembler exagérée, mais on la propose en l'état pour l'intérêt des interrogations qu'elle suscite : on peut avoir le sentiment que la linguistique et les sciences du langage se sont largement construits sur la base d'un déni phénoménologique de l'essence de leur objet observationnel, à savoir un comportement humain, un engagement moteur, une prestation biomécanique structurée. Ce déni aboutit à une conception paradoxale des langues, qui apparaissent comme des systèmes formels abstraits auto-organisés indépendemment de l'action humaine, ou relativement à une action humaine autre que celle du langage lui-même (en linguistique cognitive en particulier) ; et une conception paradoxale de la mise en parole, qui se réduit à la transcription de configurations abstraites en signaux et signes symboliques percevables à des fins de transmission et d'échange intersubjectif, la dimension interactive étant déléguée à des domaines d'études ancillaires tels que la pragmatique, la prosodie, la communication, la cogestualité. Du côté de l'enseignement des langues étrangères en France, les apprenants continuent largement à percevoir la compétence langagière comme la capacité à produire des objets phrastiques comme on produit des équations, séparant une parole théorique formalisée de l'échange communicationnel proprement dit, avec les blocages et frustrations que cela suppose en matière d'aisance, de spontanéité et d'efficacité. La perspective actionnelle vise à compenser ces limites et introduire la notion d'action, mais le primat accordé à la notion de tâche et le caractère fractionné des protocoles d'évaluation des compétences n'aboutit pas à une vision intégrée de l'expérience de l'engagement verbal interactif dans une langue donnée et rend au fond l'objet plus inabordable encore.

D'un autre côté, à l'extérieur de la linguistique, le fait que la parole soit engagement corporel est une évidence depuis longtemps, en particulier chez Marcel Jousse (2008), qui propose une anthropologie basée sur l'étude des comportements appris et leurs effets cognitifs sur les participants. Merleau-Ponty (1954) voyait dans la parole la manifestation corporelle des actes de pensée parce qu'il prenait en compte la parole intérieure, l'endophasie étudiée par Bergounioux (2004): dès lors qu'il existe des formes de pensée complexe qui pour se développer requièrent la mise en œuvre d'une simulation mentale de l'action phonatoire du corps, doublée d'une simulation des conditions de sa réception auditive, c'est que l'acte cognitif s'identifie au geste même (Lacelle 2005). Ce geste ne peut plus être pensé comme performance d'aval qui encoderait des configurations ou processus d'amont, il doit être repensé comme vecteur et manifestation des processus cognitifs reconçus comme geste mentaux (Berthoz 1997, Olivier 2012). La parole doit être reconsidérée comme un système d'action par lequelle le sujet fait advenir des expériences intellectuelles et psychologiques par 
engagement interactionnel effectif ou simulé, aussi une langue cristallise-t-elle des formes de pensée (les langages de Tlön de Borges), des modalités de l'interaction psychologique (la communication interculturelle de Ladmiral et Lipiansky 1989, la polyglossie de Tomatis 1991), et même des protocoles d'évolution des corps et voix caractérisant des communautés langagières, leur identité vue de l'intérieur et les clichés que suscitent ces apparences auprès de commautés extérieures observatrices, elles-mêmes colorées par leur propre devenir ancré dans leur action verbale. Et au-delà de ces faits généraux, la manière même dont le linguiste conçoit le lexique, la morphosyntaxe, la prosodie en tant qu'actions génératrices d'expérience immédiate et cumulée va supposer une évolution importante de la manière de décrire les faits et les interpréter. Cette reconsidération cadre avec l'un des paradigmes cognitifs actuels, l'enaction. Dans les lignes qui suivent, on en présente les principaux éléments (section 1), leur application au fait langagier (section 2), à la prononciation (section 3), à la syntaxe (section 4) et à la sémantique lexicale (section 5).

\section{Eléments de cognition enactive, perçaction et biosemiosis}

La théorie de l'enaction est née voici une trentaine d'année sous l'impulsion des biologistes chiliens Francisco Varela et Umberto Maturana (Maturna 1978, Maturana et Varela 1980, Varela et al. 1993). Le terme enaction en anglais est un nom déverbal issu du verbe enact «produire sur scène », «représenter par l'action»; ce terme est lui-même emprunté au français en acte. Le paradigme de l'enaction systématise des conceptions ancrées dans la phénoménologie du corps, de la perception et de l'action de Merleau-Ponty (1954), aussi se rattache-t-il également aux travaux de Marcel Jousse (2008), Louis Lavelle (2005), Maurice Wallon (1942), Pierre Janet (1889, 1926 et 1936), Jean Piaget (1967 et 1972).

Historiquement, l'enaction se définit comme une révolution paradigmatique supplémentaire : - à partir du début du vingtième siècle, le behaviorisme rejette le mentalisme introspectionniste comme principe et méthode d'explication des comportements observés en psychologie.

- au tournant des années cinquante-soixante, le cognitivisme, ou paradigme computationnel symbolique, issu de la cybernétique, fondé sur l'analogie cerveau / ordinateur et la distinction hardware / software (machine / logiciel) et incluant le générativisme chomskyen, se constitue en rejetant le behaviorisme et son mécanicisme.

- dans les années soixante-dix, le connexionnisme rejette la manipulation computationnelle de symboles et modélise le fonctionnement des réseaux de neurones naturels par des réseaux de neurones artificiels subsymboliques en environnement non vivant.

- au même moment, dans le domaine des sciences du langage, la linguistique cognitive américaine se constitue sur les ruines de la sémantique générative en rejetant les bases de la grammaire générative, la modularité et la computation, et avance un ensemble de théories pour lesquelles métaphores et constructions symbolisent des schèmes mentaux formalisables motivés par l'expérience biomécanique et psychologique du monde environnant (l'embodiment et l'ancrage psychologique des représentations: gestalt, catégories, schématicité).

- de son côté, la théorie de l'enaction identifie la cognition à l'action du vivant et à la relation en devenir par laquelle il s'extrait de l'environnement (Stewart 1996).

Le terme enaction "souligne la conviction croissante selon laquelle la cognition, loin d'être la représentation d'un monde prédonné, est l'avènement conjoint d'un monde et d'un esprit à partir de l'histoire des diverses actions qu'accomplit un être dans le monde » (Varela, Thomson \& Rosch 1993, 32). Concrètement, la condition du vivant est que pour toute espèce donnée, chaque corps individuel s'engage dans le monde matériel ambiant par des modalités 
propres (déplacement, actions sur les objets) et y induit des perturbations spécifiques détectables par un système sensoriel ad hoc qui permet de prélever un échantillonnage restreint des signaux ambiants disponibles. Sur cette base, la dynamique corporelle aboutit à composer une certaine mise en scène du monde, qui n'est pas l'encodage où la stylisation / schématisation d'un monde objectif extérieur qui s'imposerait aux sens comme détecteurs (et le cas échéant au cerveau comme machine à traiter l'information), mais l'invention d'un monde virtuel (comme dans les jeux video) dont la composition dépend des possibles corporels qui ont généré la perturbation des signaux échantillonnés et pertinents. Chaque être vivant «remue la bouillie ambiante » à sa manière et en rend détectable et interprétable un échantillonnage propre en fonction de ses possibilités motrisensorielles propres et compose un paysage virtuel, illusoire mais efficace, en fonction de l'échantillonnage prélevé et de son traitement.

Le paradoxe est que sans ce processus, le monde ambiant, fait de corpuscules ondulatoires, d'un champ gravitationnel quantique à boucles ou à cordes selon la théorie physique retenue, est intrinsèquement indétectable et inconnaissable : quand bien même un être vivant serait muni des percepteurs sensoriels adéquats pour capter les signaux ambiants pour ce qu'ils sont, le traitement cognitif, si poussé soit-il, n'aboutirait qu'à un effet neige analogue à celui d'un récepteur de télévision sans antenne ni décodeur, c'est-à-dire un paysage mental illisible, réfractaire à l'engagement pragmatique et hostile à la survie. En ce sens, le monde n'est pas percevable. En revanche, il est enactable: la confrontation d'un corps dynamique engagé induit des troubles locaux dont la proprioception d'un échantillonnage infime livre une sélection d'effets ramenable à une topologie schématique avec des formes, contenus, durées, distances, objets, qui ensemble font sens, exposent au sens un opéracle (théâtre d'action ouvert à l'engagement de l'observateur, par opposition à spectacle). Chez le chat et la sauterelle, l'élaboration cognitive du « haut » et du «bas » dépend de la manière dont le corps de l'animal est à même de franchir l'espace par le saut et le bond : la scène visuelle construite par le système neuronal est étalonnée par les classes de gestes mémorisés et renouvelables que le système incruste dans l'image à type d'anticipation, en sorte qu'un cerveau humain ne peut s'imaginer quel vécu apparent du haut et du bas s'imprime à la conscience de l'animal observateur, tout au plus peut-il se figurer l'orientation de la différence en s'appuyant sur l'écart entre les possibles corporels. Chez l'humain, le nouveau-né vient au monde avec un champ visuel de 30 centimètres, correspondant à la distance typique du visage de la mère pour le bébé porté à bras, et l'augmentation de la portée est appelée par l'exploration de l'espace par le déplacement et la confrontation à des objets inopinément surgis du hors-champ ; plus généralement, Piaget a montré l'ancrage moteur des facultés de perception et de cognition. La perception d'un objet tel qu'une «chaise » est une synthèse réalisant un compromis entre le traitement de signaux immédiats et l'incrustation de propriétés prédiquant des schémas d'actions possibles en fonction d'occurrences mémorisées de rencontres antérieures avec des objets analogues, en l'occurrence : chaise $=$ objet sur lequel on s'asseoit; il est tout simplement impossible de synthétiser une image sans y intégrer ces prédicats de connaissances acquis pragmatiquement, impossible de percevoir sans prédiquer, réaliser des attributions automatiques et inconscientes, résultant d'un entraînement. Pierre Janet rattachait cette faculté psychologique humaine à l'automatisme psychologique, et plus largement le paradigme de la biosemiosis se fonde sur l'idée que percevoir est sélectionner, structurer, profiler en fonction de l'expérience acquise, prédiquer en vue de faire «faire sens » à ce qui sans cela n'en aurait pas (d'où l'axiome borgésien « Le monde est un livre »).

Berthoz (1997) nomme cette condition de l'expérience la perçaction (Olivier 2012) ; dès 1934 Von Ueksküll nommait Umwelt («monde ambiant») cette enveloppe apparente spécifique que chaque espèce produit en fonction de ses moyens propres. Maturana (1978) nomme domaine consensuel d'interactions le système d'actions et d'effets rétroactifs de perception 
(perçaction) par lequel chaque espèce agit spécifiquement pour produire un monde, une figuration de l'univers ambiant (ce X-monde intrinsèquement invisible et inconnaissable) au sein de laquelle il est possible de reconnaître des espaces et objets, de s'engager, d'agir et interagir, donc de survivre. Par ce processus de la perçaction, le corps génère conjointement des effets de monde et de corps propre agissant qui fondent la relation par laquelle se constituent bilatéralement le sujet et l'objet dans la phénoménologie merleaupontyenne. Les modalités perçactives caractérisant une espèce donnée évoluent dans le temps en fonction des essais et contributions hasardeuses proposées par les individus dans les communautés de chaque espèce, faisant évoluer l'espèce corporellement et cognitivement dans une direction qui la singularise de manière croissante et la fait diverger des autres espèces animées du même devenir (processus nommé autopoïèse, fait de contribuer à l'autodétermination de son devenir par des initiatives d'origine «interne »). L'approche enactive est résolument moniste en ce qu'elle réduit la dualité corps / esprit sans céder à la tentation du physicalisme (Keller 2006), éliminant la notion de représentation dans sa version radicale (Peschard 2004); concernant le langage en particulier, elle relève de la 4-E cognition (Menary 2004 : embodied « incarné », embedded « situé », enactive «énactive », extended «étendue » par des moyens techniques ; cf. Dror et al. 2006) et de la cognition distribuée (Cowley 2009).

\section{La parole comme interface enactive}

L'approche enactive et la perçaction permettent de redéfinir l'essence du fait langagier. Parmi l'ensemble des actions dont est capable le corps humain, la parole forme un sous-système discret dont le contenu est appréhendable par ses manifestations corporelles (la phonation, l'articulation, l'audition), la modélisation des unités que l'on pense y reconnaître (morphèmes, mots, chaînes syntaxiques, variations prosodiques) et les effets sémantiques conscients qu'ils induisent globalement (le «sens » en tant que synthèse) et contributivement pour ces unités discontinues et continues (les «invariants» et leurs devenirs par mise en réseau interactif). La théorie des actes corpori-mentaux langagiers (Bottineau 2010, 2011), devenue grammaire enactive (Bottineau 2012), définit la parole comme une coordination organique exaptative (Gould \& Vrba 1982, McNeill 2005) de comportements improvisables et normés, régulés par des modèles collectifs de composition (ou d'interprétation au sens musical du terme). Parler, c'est produire, par des actes phonatoires structurés, des effets acoustiques audibles intentionnels et interprétables. Ces actes peuvent être effectivement somatisés par la voix, auquel cas la dynamique interprétative est adressée à autrui (parole « extérieure », exophasie, communication), mais ils peuvent également être simulés par le jeu de la parole intérieure (endophasie), auquel cas le bénéficiaire de l'interprétation qui en découle est le producteur lui-même, réflexivement: "parler, c'est (se) faire penser» (Bottineau 2010), soi-même ou autrui. L'inscription corporelle de la parole devient celle de la langue (Erard 1998).

Cette conception s'oppose à un encodagisme qui verrait dans les formes l'étiquetage de processus conceptuels autonomes en regard de leur affichage et publication verbale, et elle envisage la parole comme un savoir-faire corporel exécutable ou imaginable qui permet de diriger pour soi-même comme pour autrui la synthèse d'actes intellectuels construits dont la teneur diffère profondément des actes de pensée non verbale. Celle-ci réside avant tout dans la perçaction et la biosemiosis dans leur dimension automatique, à savoir la capacité à produire une scène mentale qui inclue des objets munis d'un sens hérité des interactions antérieures, lesquelles déterminent les affordances («faisabilités») de Gibson (1977 et 1979), les jugements émotionnels et attributions de valeurs, les projets pragmatiques que l'on peut en extraire en temps réel - tout cela sans parole, sans rien nommer ni planifier verbalement, 
malgré des calculs élaborés. La pensée non langagière se développe dans le rapport aux situations, espaces, objets et personnes en développant des simulations motrices de ce que pourraient être les interactions à l'espace ambiant en fonction du souvenir de classes d'engagements antérieurs avec leurs significations, intentionnalités, implications émotionnelles et intersubjectives. Par rapport à cela, l'approche enactive dégage de l'expérience de la parole les propriétés suivantes :

- comme toute action, la parole concentre le corps/esprit du sujet sur une action signifiante unifiée à l'exclusion des autres, un projet sémantique fédérateur, détournant l'attention d'un paradigme de sollicitations sensorielles non pertinentes. Par cet engagement, la parole gomme l'effet de dispersion cognitive dans le rapport au réel et «précipite » le moi en l'engageant dans la durée dans la poursuite d'un fil conducteur.

- la parole est perturbation phonatoire du monde audible (somatisée ou rêvée), c'est-à-dire couverture du monde audible par une voix audible (Berendt 1988). Parler remplace la «parole du monde » par celle du sujet, et confère la liberté d'autodéterminer les accidents perceptuels à l'origine des actes de semiosis. La parole est l'indispensable bruitage structuré par lequel l'humain dispose en tout instant de la liberté de (se) faire concevoir toute autre chose que ce qu'inspire le bruitage ambiant, par un bruitage endogène. La parole se présente comme la prise de contrôle corporelle et réflexive de la production des signaux à l'origine de la pensée, et doit être définie comme pensée verbale se substituant à, ou complétant en la transfigurant, la pensée non verbale (ce qu'avait perçu Guillaume dans les Prolégomènes avec la notion de « lucidité » et «d'hominisation », reliable à l'approche de Merleau-Ponty, avec la corporalité en moins).

- la parole est dialogique et normative (Bakhtine 1929) : les formes de l'action verbale sont des citations, des fragments de discours d'autrui (mots, morphèmes) et des enchaînements reproduisant analogiquement ceux d'autrui (syntaxe, prosodie), aussi les actes de semiosis qu'elle active sont-ils de nature sociale, conformes à des modèles schématiques partagés. Parler et penser verbalement permet d'activer des constructions intellectuelles normées par des pratiques sociales manifestées dans les interactions, telles que l'abstraction des notions lexicales, la schématicité des modèles de temporalité présentés par les systèmes verbaux et lexicaux, etc. En ce sens, la parole est un instrument qui se prothétise (Leroi-Gourhan 1964 : s'incorpore au corps humain par la pratique), modifiant bilatéralement le monde/objet et le sujet. La nature interactive de l'activité verbale, sa reproductibilité analogique, la fonde en technique commune de conceptualisation évolutive fondatrice de l'histoire (Stiegler 1994 et 2004) dont la pratique incarnée contribue à l'individuation sociale du sujet (Vygotski 1962) et de «la genèse physico-biologique de l'individu » (Simondon 1999), dont la culture (sociale) est, littéralement, naturalisée (Ogien 2007), à la fois par l'incorporation biomécanique instantanée (à chaque acte de parole) et l'enregistrement par l'espèce d'un savoir-faire développemental tourné vers l'acquisition interactive du langage (d'où la non-nécessité d'un module computationnel inné ou d'une grammaire universelle).

La grammaire enactive s'inspire donc de l'idée Merleau-Pontyenne que la conscience s'autodétermine par l'engagement corporel motri-sensoriel. Elle reprend de Bakhtine une idée secondairement présente chez Merleau-Ponty que la parole s'improvise dans le cadre d'un système de régulations collectives ancrées dans l'interaction, et permet des actes de consciences spécifiquement formatés par ces canons pragmatiques. En conséquence, elle se fixe pour objectif de rendre compte de la nature des «représentations » que le sujet peut produire dans une langue donnée en mobilisant une coordination de signifiants repensés comme boucles motrisensorielles (morphèmes, mots, chaînes syntaxiques, fluctuations prosodiques); mais au-delà de ce fait, elle assume que la parole et le langage sont une fonction biomécanique et cognitive qui constituent le sujet par l'inscription interactive immédiate et par l'entraînement récurrent, «l'expérience » : il n'est pas question de la réduire 
à l'encodage d'idées et à l'échange d'informations. La grammaire enactive converge parfois avec la linguistique cognitive pour la structure de certaines schématisations, avec la psychomécanique pour la topologie dynamique des actes de représentation, avec la théorie des opérations pour la notion de «notion» et de forme schématique; mais elle identifie la dynamique des processus mentaux à celle des processus corporels, prônant un embodiment radical du côté du signifiant (Bottineau 2011) là où la LC se contente d'un embodiment empirique, qui concerne le rapport du corps aux évènements du monde autres que le langage lui-même (on trouve bien une syntaxe de la mentalisation dans la théorie des espaces mentaux de Fauconnier 1984, mais celle-ci n'est pas directement ancrée dans la corporéité du signifiant). La grammaire enactive «merleaupontyse » la linguistique, interroge la nature de l'expérience interactive incarnée qu'est la parole, ses effets individuels, interactifs et collectifs immédiats et à long terme (De Jaegher \& Di Paolo 2007, Dinis 2010), et elle refonde son analyse des systèmes de langue sur cette conception renouvelée avec ses enjeux omnidisciplinaires : elle transforme la grammaire à l'aune de principes philosophiques, qui, d'un point de vue phénoménologique, pourraient être des évidences constituantes depuis longtemps.

Apprendre une langue, c'est apprendre une manière d'être intellectuelle, émotionnelle, interactionnelle à travers une manière de se comporter physiquement, non moins spécifique, et avec cette incidence physico-psychique immédiate et durable. La conception désincarnée des formalismes langagiers passe à côté à la fois du caractère physiquement constituant du signifiant et de la manière dont les signifiés en dépendent, la rendant incapable de rendre compte de ce qu'est l'expérience de l'engagement mono- et plurilingue, évoqué par plusieurs contes de Borges (La bibliothèque de Babel; Tlön, Uqbar, Orbis Tertius; Funes et la mémoire; Le rapport de Brodie), par l'œuvre de Vassily Alexakis (où mots et langues habitent les parleurs et les transfigurent: Mazauric 2005) et de Roy Eales (auteur de quatre recueils de poèmes multilingues concrétisés par un $\mathrm{CD}$ poétique et musical plurilingue: Bottineau 2012). On peut étudier indéfiniment la langue basque comme code et système abstrait sans jamais effleurer la question de ce qu'on fait et ce qui nous arrive quand on parle basque, de ce que l'on fait être et vivre à soi-même et autrui en engageant l'interaction selon cette modalité. Abordons cette question sous ses différentes facettes.

\section{Phonologie, phonétique et perçaction}

L'étudiant qui suit un cours de phonologie anglaise dans un cursus universitaire en France acquiert diverses connaissances théoriques concernant les paires minimales dans le système, leur réalisation contextualisées par la structure syllabique, l'accent lexical, l'accent de phrase, la prosodie..., mais il faut reconnaître que ces connaissances n'ont qu'une incidence limitée sur le savoir-faire effectif. Selon une idée répandue, seule "l'imprégnation linguistique » permet d'acquérir une capacité d'élocution effectivement mimétique relativement à tel modèle communautaire et dialectal. Ceci résulte du fait que les modèles descriptifs utilisés demeurent largement inadéquats et ne donnent pas une idée correcte de la manière dont un corps humain s'engage dans la direction vocale de l'idéation en recourant au système phonologique d'une langue donnée. Deux éléments sont en cause: l'articulation et le traitement auditif des signaux acoustiques.

\section{- l'articulation}

La production des syllabes est réalisée par une coordination de gestes moteurs qui se caractérisent par des paramétrages spécifiques qui échappent partiellement ou totalement aux modèles standards du fait même du défaut d'une approche perçactive. En français, le «mode tendu » (Delattre 1953), qui en principe relève de la phonétique corrective, consiste à réguler 
le débit de l'expiration par un contrôle musculaire continu et à faire intervenir l'articulation sur ce flux régulé. Il en résulte sa manifestation vocalique : la stabilité ou la diphtongaison limitée des voyelles, son corollaire intonatif : la limitation des glissements, ses corollaires intersyllabique: la limitation des diffusions au niveau des transitions et la réduction des inégalités entre syllabes.

Dans l'approche enactive, le système phonologique d'une langue sera défini non pas par oppositions de traits établis par l'observation des produits acoustiques et alternances, mais par coordinations de gestes articulatoires hiérarchisés. En conséquence, toute syllabe est caractérisée par le mode tendu, et toute voyelle et consonne sera définie comme une coordination de gestes articulateurs relative à une posture intégrante dénommée mode tendu, elle-même caractérisée par une posture expiratoire et musculaire soutenue. Par exemple, sans entrer dans le détail des muscles impliqués (cf. McFarland 2006), le i coordonne (i) le placage du dos de la langue au palais, (ii) un étirément latéral faible des lèvres, (iii) un abaissement limité de la mâchoire inférieur, le tout relatif à un expiration syllabique sous mode tendu. Par rapport à cela, le /y/ coordonne (i) un premier degré de rétraction du dos de la langue et, proportionnellement, un premier degré de protraction et resserrement bilabial, l'ensemble étant relatif au mode tendu. Enfin le /u/ s'obtient en procédant à (i) un second degré de rétraction dorso-lingual coordonné à (ii) un second degré de protraction et resserrement bilabial, toujours relatifs au mode tendu. Un formalisme phonologique fondé sur oppositions de coordinations articulatoires, inexistant à ce jour sous la forme que j'imagine, devrait rendre compte des contrastes entre unités phoniques par oppositions de coordinations musculaires actives, corrélées à leurs manifestations sensorielles multimodales du point de vue des expérients relationnels impliqués, à savoir: (a) ce que ressent le producteur par proprioception auditive et tactile, (b) ce que perçoit l'auditeur-observateur par perception auditive et visuelle (pour la partie visible de l'articulation), (iii) ce qu'observe et modélise l'analyste scientifique techniquement équipé (les positionnements des articulateurs, en gros ce dont rendent compte les traits dans leur conception traditionnelle). Une telle phonologie perçactive subordonne la phonologie au mode phonétique (mettant un terme à la distinction) et se donne les moyens de rendre compte des gestes productifs et des effets sensoriels intersubjectivement distribués, donc, corollairement, du potentiel biosémiotique interprétable par chacun à partir de l'expérience vécue de l'articulation.

Considérons l'anglais à titre de contraste. L'anglais se parle sur un mode que l'on peut qualifier d'expiratoire, qui contraste radicalement avec le mode tendu : la posture de base, à l'inverse du français, consiste à laisser le débit de l'expiration profiler la structure dynamique des syllabes. Ceci se traduit par un débit intrasyllabique décroissant d'un maximum en début de syllabe vers un minimum en fin de syllable: One! Two! (qui ne doit surtout pas être réalisé [tuuu] en mode tendu français). Pour le système phonologique, il en résulte (i) des voyelles longues faiblement ou fortement diphtonguées, toujours fluctuantes, sauf si leur glissement est bloqué par une consonne dans la coda syllabique (put); (ii) en attaque syllabique, des consonnes plus fortement plosives que celle du français, au point d'être disjointes de la voyelle par une «fuite » pour les non voisées (tea [ $\left.\mathrm{t}^{\mathrm{h}} \mathrm{i}:\right]$ ), sauf si la pression expiratoire est initialement réduite par une sifflante précédant la plosive (sting) ou bloquée par un coup de glotte (américain day/d $\varepsilon /$ ). En conséquence, le /u:/ long de l'anglais se forme (a) en faisant coulisser le point de placage dorso-vélaire d'un point correspondant approximativement à celui de /y français vers l'arrière, si bien que la voyelle finit par sonner par /u/, (b) en s'abstenant d'opérer la protraction-resserrement bilabiale du second degré que le $/ \mathrm{u} /$ français corrèle à la rétraction, (c) en synchronisant le coulissement arrière avec la décroissance de la pression expirée. La terminologie est inadéquate en ce que parler de voyelle tendue pour l'anglais est un contresens, il s'agit au contraire de voyelle « détendues » ou «décontractées » qu'on laisse se produire en se soumettant à une expiration appuyée et 
décroissante, d'où en proportion un glissement intonatif (que l'on retrouve en italien par opposition à l'espagnol sur les «mêmes» mots). La prépondérance de l'expiration sur l'articulation induit des affrications (Tuesday prononcé «chooseday»), des diffusions par assimilation (this year prononcé thish year, et même, avec franchissement d'une plosive : next year prononcé "neksht year», très courant), et des alternances entre syllabes activement «appuyées » (accentuées) et syllabes inertes ou passives (inaccentuées). La qualité des voyelles anglaises et même le système d'oppositions dépendent entièrement de la manière dont chacune est une coordination gestuelle d'articulateurs appliquée à un mode vocalique qui est soit actif (appuyé, accentué), soit passif (inaccentué), avec diverses pondérations relevant (i) de l'accent syllabique au sein de la séquence formant le mot (mono-, di-, trisyllabique..., avec l'accent initial, médial ou final, éventuellement un accent primaire et un secondaire) et (ii) de l'ajustement du schéma accentuel lexical à la dynamique phrastique intégrante (nom, adjectif, verbe, position dans l'énoncé ; accents contrastifs ou emphatiques).

Pour un francophone, parler en anglais suppose avant tout une inversion hiérarchique des coordinations mises en conflit : là où en français l'articulation régule l'expiration, en anglais c'est l'inverse qui se produit, et tout le reste en dépend (phonologie, phonétique, rythme, intonation). Parler en anglais avec le mode tendu donne le stéréotype du français qui croit parler en anglais (cf. le titre du best seller God save la France : A Year in the Merde de S. Clarke, 2005); parler en français en mode expiratoire livre le parler asthmatisant de Jane Birkin - avec, dans les deux cas, un dégagement de connotations psychologiques et culturelles correspondant aux modes d'engagements corporels respectifs. Et plus crucialement, l'engagement régulier d'un corps dans un mode phonatoire donné fait évoluer ce corps avec le vieillissement dans une direction orientée par les contraintes inhérente à ce mode, ce qui est une forme d'individuation autopoïétique du corps biologique individuel dans le cadre des rapports sociaux où il s'inscrit et qui le contraignent. L'anglais américain se caractérise par un degré de projectivité des syllabes accentuée et de variation des degrés d'aperture qui rendent les voix extrêment sonnantes (dans les stations de métro parisien, une observation attentive montre que les voix les plus portantes sont les américaines, pas les italiennes, contrairement à ce que laisserait prévoir le cliché) et aptes à monter dans les aigus (ce qui se remarque chez les hommes, cf. la voix de Woody Allen dans ses conflits avec Mia Farrow) comme à descendre dans les basses (ce qui se remarque chez les femmes, en particulier de petite taille, cf. Rosanna Arquette). En arabe algérien et lybien (cf. la voix de Kadhafi), en italien napolitain et sicilien (cf. la voix du Parrain), en espagnol (surtout andalou), divers conflits opposant l'articulation au mode syllabique contraignent la voix au point d'occasionner des sortes de dégénérescence (timbre rauque du chanteur de flamenco pour l'espagnol, que l'on retrouve chez les actrices des films d'Almodovar - même si elles ne fument pas). Par suite, l'apprentissage d'un mode phonatoire étranger à un âge avancé (collégien, lycéen) suppose le réengagement des corps dans une discipline phonatoire sportive pour laquelle le corps n'a pas été préparé et dont l'esprit n'est pas conscient, sans compter les blocages psychosociaux, puisque cet engagement revient à accepter de jouer la comédie sincère de l'identité corpoculturelle de l'autre et, plus profondément, à rejouer la relation d'identification au corps de la mère par le biais de l'amalgame attitudinal (largement médié par les neurones miroirs). Cette dimension corrective ou remédiative, bien perçue par la phonétique applicative du même nom, a confiné cet aspect du problème aux études orthophoniques et phoniâtriques, mais l'a fait évacuer des phonologie et phonétique théoriques, et même de l'enseignement des langues, alors même qu'une orthophonie contrastive serait indispensable tant pour la formation initiale des enseignants que pour la pratique quotidienne des apprenants.

Considérons le cas de l'espagnol castillan, qui pour le francophone pose entre autres les difficultés du r roulé, du s chuinté, du t/d intervocalique spirantisé. Le mode caractérisant le castillan est ce que je nomme un peu ludiquement le mode «prognathique », à savoir, le fait 
que l'attitude phonatoire de départ, neutre, au repos et non marquée (cf. le visage ovale des nobles dans le tableau de El Greco El Entierro del Conde de Orgaz à Tolède ${ }^{1}$ ) suppose un positionnement légèrement avancé de la mâchoire inférieure, plus ou moins visible selon les personnes, mais suffisamment actif pour reconfigurer tout le système articulatoire et affecter très perceptiblement le vieillissement de l'appareil phonatoire. Ce léger prognathisme a pour effet (i) d'appuyer l'apex contre la couronne (d'où l'écrasement des $t$ et $d$ ), voire de le lui faire dépasser (d'où la post-dentales $\mathrm{z}$ 《 ceta », qui en castillan résulte du prognathisme et non d'une projection musculaire active comme en anglais: cette différence articulatoire fondamentale conditionne la différence audible et la signification biosémiotique du geste pour les percepteurs ; une description non motrice des phonèmes anglais et espagnol aboutit à des traits convergents et passe à côté du différenciel musculaire, ce qui est grave). Ce prognathisme suppose également que pour produire une apicale non plosive, non plaquée contre la couronne, il est nécessaire de réaliser une rétraction linguale pour compenser l'espace oral perdu sous l'effet de l'avancement, d'où (i) la mouillure du s, légèrement palatalisé, (ii) la vibrance du r roulé (obtenu par vibration libre de l'apex en fin de langue rétractée), (iii) la résonance en fond de gorge (très audible avec les $\mathrm{u}$, o et a, et les nasalisations). Pour s'en convaincre, que le lecteur tente l'expérience de recaler sa mâchoire inférieure dans le sens indiqué (prognathisme), puis à parler en français en s'imposant de maintenir cette posture forcée : si le geste est correctement réalisé, il doit déterminer en français le roulement du $r$, le chuintement du s, l'écrasement de $t$ et $d$, le blocage de la protraction bilabiale de degré 1 (l'impossibilité de former un /y/), la déformation des nasales, etc. L'exercice est difficile parce qu'il impose au corps vocal «francisé » un exercice pour lequel il n'est pas préparé physiquement, mais que réalise inévitablement le corps vocal « hispanisé » d'un vieil Espagnol émigré de la Retirada (l'exil consécutif à la guerre civile de 1936-39), qui conserve généralement son «accent» après des décennies de vie en France conserve, en fait, le mode phonatoire prognathique auquel son corps s'est adapté et qui conditionne toute la production, avec les effets biosémiotiques et acculturants que cela suppose.

Ainsi, les modes phonatoires des langues cristallisent des styles motrisensoriels et systèmes phonologiques qui font évoluer les corps dans des directions déterminées et profilent des apparences collectives qui ne manquent pas d'être repérables par différenciation et par contraste du point de vue des regards portés par des observateurs appartenant eux-mêmes à d'autres communautés, elles-mêmes physiquement profilées (à leur insu) par les tendances générales de leur propres langue ; il s'ensuit l'émergence des clichés culturels évoqués, en fait motivés par des faits linguistiques et éthologiques structurants qui n'ont pas à ce jour fait leur entrée dans la linguistique malgré leur rôle crucial dans la formation des systèmes, et que l'approche enactive et perçactive est à même de constituer en objet d'étude scientifique.

\section{- l'audition.}

Selon la théorie motrice de la perception auditive (Mattingly \& Libermann 1985), l'audition et la reconnaissance des sons langagiers ne consiste pas à catégoriser un phonème ou une syllabe par identification de traits acoustiques, mais à opérer une simulation neuronale du geste moteur par lequel le percepteur produirait lui-même le son articulé. Illustrons ce fait par

http://www.google.fr/imgres?um=1\&hl=fr\&tbo=d\&biw=1680\&bih=812\&tbm=isch\&tbnid=gvTm4TruWxIE6M :\&imgrefurl=http://es.wikipedia.org/wiki/El_entierro_del_Conde_de_Orgaz\&docid=GhP53ehLM-

A5ZM\&imgurl=http://upload.wikimedia.org/wikipedia/commons/thumb/1/19/El_Greco_-

_The_Burial_of_the_Count_of_Orgazdetal1.jpg/600px-El_Greco_-

_The_Burial_of_the_Count_of_Orgazdetal1.jpg \&w=600\&h=265\&ei=o6XJUNrAOcOHhQfIgoHQAw\&zoom $=1$ $\&$ iact $=$ hc \&vpx $=103 \& v p y=147 \&$ dur $=66 \&$ hovh $=149 \&$ hovw $=338 \&$ tx $=123 \&$ ty $=105 \&$ sig $=1085080088610404658$ 20\&page $=1 \&$ tbnh $=135 \&$ tbnw $=270 \&$ start=0\&ndsp=56\&ved=1t:429,r:1,s:0,i:83 
l'effet McGurk, identifié par une expérience déjà ancienne (McGurk \& McDonald 1976). On fait entendre à un sujet la syllabe «ga » dans des écouteurs tout en lui présentant à l'écran la video d'un personnage prononçant «ba » en synchronisant parfaitement les signaux audio et video, faisant croire que le son entendu correspond à la phonation observée visuellement, et on demande au sujet ce qu'il entend. Contre toute attente, la plupart des sujets testés répondent « da », une syllabe ne correspondant ni au signal audio, ni au signal video. Il existe plusieurs interprétations du phénomène, qui a donné lieu à une série continue de recherches depuis l'époque; l'une d'entre elles, étayée récemment par des expériences réalisées par Olivier et Bottineau (à paraître), consiste à dire que la reconnaissance d'une syllabe passe par la simulation d'un geste moteur habituel, et qu'en cas de conflit généré artificiellement, le système cognitif est contraint de choisir un geste de compromis : ici, le contraste dorso-vélaire / bilabial est neutralisé par la simulation du geste médian, apico-dental, ce qui se traduit par la production d'un effet conscient de type «da», preuve que dans l'émergence du son à la conscience, la simulation motrice l'emporte sur le décodage calculatoire des caractéristiques audibles de l'évènement acoustique.

Le corollaire est que chez l'apprenant d'une langue seconde, l'incapacité à produire le geste moteur pour certains sons induit l'incapacité de réaliser la simulation gestuelle mentale requise pour la production consciente du son émis par autrui, ce qui mène à une forme de surdité sélective. Ceci se vérifie abondamment chez les francophones dans le cadre d'exercices de compréhension en anglais, et j'ai pu tester en cours (en école d'ingénieurs) le fait que la prise de conscience d'une motricité ajustée entraîne effectivement une amélioration rapide des performances en compréhension. La théorie des neurones miroirs de Rizzolatti et Craighero (2004) ne dit pas autre chose : chez le singe, des neurones homologues de la zone F5 sont coactivés parallèlement chez l'individu mangeant une banane et chez l'individu observant le premier, comme si la compréhension de la scène passait par la simulation d'un geste inhibé ; chez l'humain, les zones correspondantes sont les aires de Broca du contrôle moteur de la phonation, coactivées chez le phonateur et l'auditeur. L'effet de surdité s'éprouve également lorsque l'apprenant, produisant correctement un geste vocalique (cas de certaines voyelles du russe), émet un son qu'un auditeur natif identifie comme correct et conforme au modèle alors même que l'apprenant français ne s'entend pas prononcer correctement cette voyelle (pas plus qu'il ne la reconnaît chez son modèle, ceci m'est arrivé à diverses reprises): ce phénomène curieux se produit en phase intermédiaire lorsque l'apprenant parvient à improviser une imitation correcte du geste articulatoire visuellement observable mais que, par manque d'entraînement et d'autonomie, il n'a pas encore construit et enregistré un modèle neuronal de la coordination gestuelle en question, ce qui le rend incapable de simuler aussi bien le son que lui-même produit que celui qu'il a entendu en modèle. Même quand on en connaît les ressorts, le phénomène est déconcertant, et il démontre l'inévitabilité de la simulation motrice dans la production enactive d'une présentation sonore à la conscience. De la même manière, l'effet McGurk est spectaculaire dans la présentation qui consiste à présenter à l'écran en parallèle les visages disant «ba » et «fa » alors que le signal audio est unique, «ba » : ce qu'entend le spectateur dépend, de manière convaincante, du visage sur lequel il focalise son attention, faisant varier l'effet auditif « perçu » avec la cible visuelle choisie alors même qu'il sait que le signal audio répété est constant ${ }^{2}$. Les implications d'une telle approche sont considérables en matière d'acquisition d'une langue première et d'apprentissage d'une langue seconde, et de telles expériences militent fortement en faveur du paradigme de la cognition gestuelle, à savoir l'idée que tout acte cognitif, y compris verbal, passe par l'exécution ou la simulation d'un geste moteur, chez le locuteur comme chez l'auditeur ou le lecteur et le penseur.

\footnotetext{
${ }^{2}$ http://www.youtube.com/watch?v=G-1N8vWm3m0
} 


\section{Syntaxe}

En grammaire enactive, les structures ou constructions syntaxiques sont redéfinies en y incorporant le facteur temps, à savoir l'importance de la succession chronologique des éléments énoncés, le rôle de leur moment de survenance dans le processus d'élaboration du sens, la manière dont les éléments interprétés sont reprofilés avec l'arrivée des éléments ultérieurs. Cette approche caractérise déjà la chronosyntaxe de Macchi (1986), la théorie des formes en sémantique de Cadiot et Visetti (2001), la grammaire instructionnelle de Col et al. 2010, et plus anciennement les propositions de certains guillaumiens en syntaxe comme Valin (1981), Le Flem et Cotte (1996), mais elle s'articule ici avec la conception enactive du signifiant. La syntaxe sera définie comme le protocole habituel de construction du sens dans l'exercice du rapport interlocutif. L'objectif est de définir les enchaînements récurrents qui caractérisent les périodes énonciatives, les tirades monologales et les répliques dialogales, dont la formalisation graphique produit les objets nommés «phrases ».

A titre de comparaison, on trouve des exemples de tels cycles récurrents en mécanique : pour le moteur à explosion, l'enchaînement admission / compression / explosion / échappement ; en biomécanique : pour la respiration, la séquence inspiration / expiration ; pour le rythme cardiaque, la séquence systole / diastole. Ces unités d'enchaînements se présentent comme des tirades munies d'une syntaxe récurrente avec des formulaires enchaînant des gabarits porteurs de morphèmes dont on comprend l'effet en en étudiant leur profil dynamique dans le contexte où il opère. De même que les cycles réguliers de la respiration et de la cardiovascularité rythment le métabolisme corporel, la répétition des séquences syntaxiques, dont les unités varient diversement mais sont sous-tendues par des schèmes de base tels que la relation sujet / prédicat, forme des cycles énonciatifs et une horloge cognitive qui vont former et rythmer l'activité psychique sous contrôle verbal selon des schémas d'action récurrents pour le sujet et intersubjectivement fédérateurs pour les interactions. En typologie générale, on identifie différents modèles d'horloges syntaxiques, munies d'une structure narrative spécifique (comparable à la morphologie du conte chez les formalistes russes), et ces modèles singularisent la conduite de la formation et même son contenu dans le cadre des rapports intersubjectifs, eux aussi gérés selon les formats de ces enchaînements. Considérons les cas du basque et du breton.

\section{L'horloge syntaxique du basque}

Le basque est une langue ergative avec phrases à verbe final avec accord personnel multiple à un, deux ou trois arguments (voire quatre, en cas de conjugaison allocutive et « ditransitive »). La procédure consiste à d'abord former séparément un certain nombre de participants lexicaux, puis à les regrouper dans le verbe : Peiok andreari liburua emango dio «Peio donnera le livre à sa femme ».

Chaque argument (Peiok, andreari, liburua) est formé en suivant un protocole commun, à (i) savoir la formulation du participant notionnel par une lexis (Peio, lore, andre) et (ii) l'attribution à ce segment d'une fonction relationnelle par une série de marqueurs agglutinés formant le nexus ( $-a$ pour la relation notion / occurrence: la détermination; d'autres marqueurs pour les rôles sémantiques). La lexis est dans cet exemple lore- $a$ réduite à un élément lexical, mais elle pourrait être plus étoffée (y compris une proposition non finie ou finie). L'ensemble d'arguments de forme lexis + nexus précède généralement le verbe, et l'on y distingue les actants qui déclenchent l'accord verbal (ceux à l'absolutif, à l'ergatif et au datif) des non-actants ou «inactants» qui ne le déclenchent pas (inessif pour une localisation : etxean «à la maison», allatif pour une destination : etxera «à la maison», 
ablatif pour une origine etxetik: «de la maison », instrumental pour une manière autoz: «en auto », partitif pour une quantification dirurik: «d'argent» après négation, avec de nombreuses surcompositions (jusqu'à quatre niveaux, pour des fonctions complexes : etxerakoan 'dans le d'à la maison' «en allant à la maison »).

Concernant les actants, on relève un cas zéro, l'absolutif, pour l'argument pris pour objet de discours, et deux cas morphologiquement marqués, l'ergatif $-k$ (cf. Peiok) et le datif $-i$ (cf. andreari). Ces deux marqueurs spécifient la hiérarchisation de ces arguments complémentaires par rapport à l'argument principal: l'ergatif marque une relation de supériorité, de domination ou d'intervention, et fait typiquement de l'argument lexical l'agent relatif à un patient ; inversement, le datif marque une relation d'infériorité, de résultativité ou de destination, et fait de son argument le destinataire ou affectataire du procès (bénéficiaire, détrimentaire, expérient). L'interprétation de l'absolutif non marqué et non polarisé dépend à la fois du sens du verbe (agentif, situatif...) et des relations avec d'éventuels autres arguments (ergatif et/ou datif), ce qui peut lui faire prendre des valeurs d'agent (de verbe « intransitif »), patient (de verbe «transitif», trajecteur (de verbe destinatif « ditransitif»), etc. : l'absolutif neutre en matière de polarisation varie de manière plastique en se reliant d'un côté à d'autres arguments et de l'autre à un verbe (cette approche typiquement chronosyntaxique clarifie la dynamique de l'interprétation, alors que l'approche traditionnelle par les correspondances forme / sens, les alignements de Dixon 1974, et les structures de valence «plates » type intransitivité, transitivité, ditransitivité, obscurcissent le tableau et ne rendent pas justice à la simplicité des processus en cause). Par cet appareil morphologique de type casuel, l'énoncé basque construit ainsi une matrice actancielle directement entre arguments nominaux, sans passer par le verbe, lequel n'est pas le centralisateur actanciel imaginé par Tesnière 1959 pour les langues romanes. Cette matrice actancielle est de type gestaltique : elle discrimine une base (l'absolutif) et deux saillances, l'une en pic d'instabilité (l'ergatif), l'autre en creux de stabilisation (le datif), entre lesquels elle définit un profil relationnel dynamique schématisé et générique. Cette valeur différenciatrice de l'ergatif et du datif relativement à l'absolutif explique l'impossibilité de coréférence entre arguments sous cas distincts et bloque l'apparition de structures réfléchies dans les double et tripleaccords quand bienmême elles sont morphologiquement générables, d'où le recours à des tours lexicaux pour le réfléchi bere buru- «sa tête » et le réciproque elkar «mutuellement », bat beste- «l'un l'autre » (Bottineau \& Roulland 2007).

Une fois cette première partie réalisée en chronosyntaxe, les arguments lexicaux et leurs rapports dynamiques sont connus. La seconde partie introduit le verbe, lequel va incorporer par agglutination des indices morphologiques qui renvoient à chacun de ces arguments, ce qui donne lieu à des accords simple, double ou triple selon le nombre et type d'arguments impliqués (absolutif, absolutif + ergatif, absolutif + datif, absolutif + ergatif + datif, triple dans le cas de dio « il le lui »). Le jeu est complexe parce que le verbe reçoit également des marqueurs de temps ( $-n$ pour le passé, même marqueur que pour l'inessif de localisation et le possessif personnel) et de modalité (-ke pour l'hypothèse, à rapprocher de -ko des participes futurs et des possessifs conceptuels), et que les infixes personnels varient en forme et position en fonction de la cooccurrence d'autres infixes de personne et des paramétrage temporels et modaux : dio au passé devient zion et non *dion, au conditionnel lioke et non *dioke, etc. L'esprit du système est que chaque argument est retraité dans l'environnement actanciel verbal en fonction de l'état général du réseau formé par les rapports avec les autres actants et les paramétrages de temps et d'aspect, ce qui fait de la morphologie verbale un instructeur explicite de l'état d'un réseau, phénomène que l'on ne connaît pas dans les langues romanes. Par exemple, «moi, j'étais » se dit ni nintzen, où moi est dit une première fois sous la forme d'un pronom absolutif $n i$, puis est repris dans l'agglutination sous la forme nin (ni augmenté d'une marque de passé $-n$ ) alors que le reste de la composition est en fait un passé de 
troisième personne («était ») : «moi » est pensé deux fois, l'une au présent de parole pour le je présent du locuteur, l'autre au passé qu'il s'agit de faire imaginer pour le même locuteur pensé hors du présent d'interlocution et engagé dans le passé narratif. La discrimination de l'argument lexical et de l'agglutination verbale fait en sorte que la structure impose explicitement à ses usagers interlocutifs de conceptualiser deux fois «moi » sous ces deux instances contrastées, fournissant une notice de montage très explicite du protocole métalinguistique de production du sens. Une modélisation enactive de ce type de structure y voit non pas l'encodage d'une structure conceptuelle schématique d'origine psychologique et non langagière, mais un protocole verbal par lequel des formes signifiantes motri-sensorielles imposent à l'interprétation une procédure routinière de déploiement du sens. Cette procédure existe en basque parce qu'elle est efficace et que les usagers en ressentent l'efficacité, donc la conservent dans la reproduction analogique des formes d'un exemplaire au suivant ; elle n'est pas pour autant motivée, et encore moins déterminée, par des impératifs psychologiques non langagiers qu'elle serait tenu d'épouser et refléter (sinon, toutes les langues épouseraient ce modèle).

L'agglutination peut en basque porter sur le radical du verbe: badaramakiot «je le lui apporte », baneramakion «je le lui apportai ». cette conjugaison dite synthétique concerne une douzaine de verbes fréquents ; les autres verbes présentent une conjugaison analytique de forme participe préfinal + auxiliaire final (cf. eman dio), où le participe reçoit des affixes aspectuels correspondant en gros à l'infinitif (emango), au gérondif (ematen) et au participe passé (eman) des langues romanes, alors que l'auxiliaire (dio) reçoit les marques de personne, temps et modalité (passé nion, conditionnel nioke) délocalisées du verbe, mais selon les mêmes principes combinatoires, variationnels et agglutinatifs. L'aspect du participe et la matrice de l'auxiliaire varient séparément et librement, de manière modulaire, ne formant pas les périphrases verbales corrélatives de type avoir + participe passé (vs *avoir + infinitif / gérondif), ce qui rend le système basque très productif, générateur de nuances aspectuelles et modales inconnues du français. Au niveau de l'accord, l'énoncé canonique reproduit spéculairement la même matrice du côté des nominaux explicites ou implicites en amont et du côté de l'agglutination (verbe ou auxiliaire) en aval, mais il est possible de « désharmoniser » les deux niveaux soit en ajoutant au verbe des marqueurs imprévus en amont, comme celui de l'allocutaire masculin ou féminin à qui l'énoncé est destiné : Peiok andreari liburua eman ziok/zion «(je te dis à toi homme / femme que) Peio a donné le livre à sa femme ». L'effet de sens est, selon les contextes, une parole intime dans un couple, une invective dans un conflit, la révélation d'un secret, ou l'affichage en public d'une relation privilégiée - autant de faits pragmatiques que le français ne grammaticalise pas et qui déterminent en basque une gamme d'interactions psychosociales qui nous sont inconnues sous ce type de formes, sauf avec le datif éthique (cf. entendu à Marseille lors d'un altercation dans un café Oh! Celui-là, je m'en vais te me le désosser!). De même, l'occultation d'éléments dans l'accord multiple produit le passif et surtout les antipassifs, comme Andrea liburua emanda da 'le femme le livre donné est', 'la femme est dans l'état d'avoir été donné le livre' «la femme a reçu le livre » (implication : elle est dans l'état résultant de l'action, par exemple satisfaite). Cêtte structure discrimine deux niveaux de prédication (da «est» et emanda «donné »), et chaque absolutif est relié séparément à son propre verbe (andrea à da, liburua à emanda), d'où l'absence de marquage datif du destinataire (andrea n'est pas relié directement à liburua, pas plus que emanda da ne forme un couple participe + auxiliaire d'un verbe unique).

La subordination s'appuie sur les mêmes principes que précédemment: elle consiste à enchâsser une proposition à verbe final fini ou non fini dans un rôle de lexis clôturé par un nexus relationnel, c'est-à-dire l'une ou plusieurs postpositions du types de celles qui s'applique au nom. L'inessif (etxean « à la maison») forme les temporelles (etorriko da «il viendra », etorriko denean «quand il viendra ») en «inessivant » une proposition entière, le 
génitif (etxearen « de la maison ») forme les relatives (ezagutzen dugun etxea « la maison que nous connaissons »). Ce dispositif récursif permet des formulations compactées «impensables» dans une langue romane : Zer garelakoan zaude? 'vous êtes dans quoi que nous sommes' «pour qui nous prenez-vous? », [questions d'un journaliste à un auteur basque] Gaur egun zer ez ikusteak ematen dizu pena? «Aujourd'hui, ne pas voir quoi vous donne de la peine? » (où la question zer ez ikuste 'quoi pas voir' est à l'ergatif déterminé -ak, relié à l'absolutif pena «peine » et accordé à l'auxiliaire dizu avec triple accord «il vous le » du participe présent ematen « donnant»), Eta zer ez ikusteak ez dizu faltarik egiten? «Et ne pas voir quoi ne vous manque pas ? (avec une négation $e z$ dans la subordonnée et une autre dans la principale); réponse: Gure arteko elkar ulertzerik ez ikusteak. 'Ne pas voir de compréhension entre nous.' « Ne pas nous voir communiquer entre nous en basque » (avec le nom verbal ulertze «comprendre » et toute sa proposition au partitif -rik entraîné par la négation, cf. ez dizu faltarik egiten 'ne vous fait pas de faute' "ne vous manque pas » avec le même partitif négatif de portée nominale). Si on croit que les constructions encodent des structures conceptuelles, on se dit que les Basques sont des gens bien compliqués, à qui il vient spontanément à l'esprit des écheveaux abscons que le discours et la grammaire reflètent. $\mathrm{Si}$ on se dit au contraire que la morphosyntaxe, redéfinie comme une discipline perçactive et un domaine conventionnel d'interactions enactives, est une gymnastique comportementale incarnée porteuse d'une gymnastique intellectuelle associée et interactive, une technique cognitive incarnée, alors on comprend que l'entraînement au potentiel créatif du système développe naturellement un savoir-faire cognitif afférent à ses possibilités constructionnelles, comme le montrent ces questions étranges, improvisées dans le dialogue en temps réel avec une spontanéité et une aisance déconcertantes, sans snobisme aucun, par un journaliste au cours d'une interview en direct à bâtons rompus.

\section{L'horloge syntaxique du breton}

L'énoncé breton se caractérise par un verbe ou auxiliaire conjugué en deuxième position, séparant un constituant initial focalisé des autres constituants non focalisés. La focale initiale peut être le sujet, l'objet, l'attribut, un circonstant, le verbe lui-même (à une forme non conjuguée, infinitive ou participiale, l'auxiliaire conjugué étant en deuxième position). Ce dispositif donne au locuteur le choix de commencer l'énoncé par le constituant essentiel, celui qui motive la prise de parole et par lequel il entend «impacter » la conscience de l'allocutaire. «Je m'appelle Didier» se dit Didier eo ma anv 'Didier est mon nom', «Il fait froid » se dit Yen eo an amzer 'froid est le temps', "Voulez-vous du café ? » se dit Kafè az po 'café votresera ?', et ainsi de suite (cf. la syntaxe de l'anglais du personnage de Ioda dans la saga Star Wars qui, en anglais comme dans la traduction française, calque ce type de syntaxe à initiale focalisatrice : «Révélée ton opinion est ! »). Pour tout énoncé, il y a moyen de produire autant d'allostructures que de choix de constituants possibles à l'initiale : Didier eo ma anv 'Didier est mon nom' focalise Didier, mais on pourrait dire Ma anv a zo Didier 'mon nom est Didier' pour souligner que Didier est mon nom (et pas autre chose), et des énoncés plus riches offrent de nombreux choix de focalisations possibles.

Dans ce système, la fonction du syntagme initial n'est précisée ni par la position (qui détermine la focalité, rien de plus), ni par la morphologie, puisqu'il n'existe pas de marque casuelle lexicale de type latin (nominatif, accusatif etc.). De ce fait, l'attribution d'une fonction à la focale est gérée par un autre dispositif: (i) une particule préverbale instruit l'interprétant soir de connecter la focale au verbe (préverbe $a$ ), soit de ne pas le faire (préverbe $e$ ), ce qui fait de la focale un actant (sujet ou objet) ou ne le fait pas (attribut ou circonstant). (ii) le verbe lui-même reçoit une flexion personnelle de sujet si la focale est autre que le sujet (ul levr a lennan 'un livre lis-je' «je lis un livre»); si le sujet est focalisé, le verbe marque le temps, mais pas la personne (ce qui évite de convoquer le sujet dans deux 
positions contradictoires, focale et non focale : me a lenn_ul levr 'moi li_ un livre' «je lis un livre »). En chronosyntaxe, l'élément initial est compris comme focal, puis il est incorporé à la valence verbal par le préverbe $a$, puis il devient sujet ou objet au moment où le verbe est formulé, porteur d'une flexion sujet qui donne réotractivement à la focale le rôle d'objet, ou dépourvu de cette flexion, laissant rétroactivement à la focale le rôle de sujet. Ainsi, on ne peut dire que la focale «est» le sujet ou l'objet (ou autre chose selon les exemples), mais qu'elle le devient à mesure que progresse l'énonciation avec des marqueurs qui éliminent des indéterminations (conformément au modèle culiolien). Par ce dispositif, le choix de la focale est libéré, puisqu'il sera toujours possible de lui attribuer a posteriori un rôle syntaxique par le biais d'une succession d'autres marqueurs rétroactifs, et ce dans une langue qui ne fixe la fonction ni par la position, ni par la forme (en l'absence de morphologie casuelle). Cette dynamique d'attribution rétroactive de la fonction ouvre également la possibilité de subordinations sérielles sans conjonction ni pronom relatif, comme Me a wel ul levr a zo war an daol 'moi voi_ le livre est sur la table' «moi je vois le livre qui est sur la table » où ul levr «le livre », objet de wel « vois », est réinterprété comme sujet de zo « est » par l'intermédiaire du préverbe d'incorporation actancielle $a$, ce qui a pour effet d'attribuer à $u l$ levr deux fonctions successives en temps réel en deux moment consécutifs de l'énonciationinterprétation, d'où le non-rejet de la double fonction et l'inutilité du pronom relatif qui la résoudrait. Le français ne connaissant pas cette attribution progressive de la fonction, il ne connaît pas non plus sa renégociation, d'où *Je vois le livre est sur la table, où le livre apparaît muni de deux fonctions adversatives synchronisées à l'interprétation, problème que résout le relatif qui en redupliquant la même entité notionnelle en deux moments linéaires successifs et discrets où les fonctions peuvent être attribuées en parallèle, séparément et sans conflit cognitif.

Cette dynamique sous-tend toute la grammaire bretonne: indépendantes, relatives, conjonctives, sujets disloqués tels que Per a zo brav e ti 'Pierre est beau sa maison' "La maison de Pierre est belle » ou «Pierre a une belle maison », où la focale Per forme un sujet focalisé provisoire en attente de spécification déterminative tardive par $e t i$, ce qui permet de le focaliser séparément sans y inclure l'objet possédé. Apprendre le breton suppose donc l'acquisition d'une double gymnastique, celle des signifiants par laquelle on réalise des attributions progressives de fonctions et des redéfinitions notionnelles en temps réel (mécanisme inconnu en basque comme en français), et celles des signifiés par laquelle on amorce la parole par la composante efficace pour l'interprétant (allocutaire adressé ou locuteur endophasique réflexif), ce qui suppose que la langue entraîne ses usagers à une théorie de l'esprit de soi ou de l'autre, engrammée dans les protocoles syntaxiques.

Pragmatiquement, en breton, on ne commence jamais une phrase sans que le choix de l'amorce focale ne soit motivé par le rapport intersubjectif à l'autre (ce qui en français n'est le cas qu'à l'oral, avec les détachements topicalisés : mon frère, sa moto, le guidon, le chrome, il est parti). La syntaxe bretonne requiert une «étreinte psychologique interactive » particulière entre interlocuteurs (Bottineau 2012), fait qui a été décrit en ethnopsychiatrie (Denez 1986), et dont les bretonnants eux-mêmes reconnaissent faire l'expérience quand ils parlent sur les blogs du plaisir qu'ils éprouvent à vivre le rapport à l'autre en breton plutôt qu'en français. On constate ici que l'horloge syntaxique d'une langue ne concerne pas uniquement l'ordination d'opérations qui ne varieraient pas dans leur contenu d'une langue à l'autre ; au contraire, chaque langue fait vivre à ses usagers une expérience psychosociale particulière qui n'est pas réductible à celle des autres langues. La langue utilisée n'est pas innocente dans la formation de la psychologie de l'individu pour ce qui est de ses facettes sociales et interactives.

\section{L'horloge syntaxique du français}


L'énoncé français est ramenable à un couple SN / SV dans cet ordre (Je dors, il pleut), lequel peut être étoffé par (i) l'expansion du SN (par des GN, des subordonnées non finies et finies), (ii) celle du SV (par la complémentation verbale et divers ajouts non actanciels), (iii) par diverses étapes supplémentaires, introductrices ou interpolées (les détachements présentatifs et topicalisations, les appositions insérées). Le couple de base SN / SV est polyfonctionnel ce que sa succession articule (a) une hiérarchie gestaltique avec une entité prise pour fond ( $\mathrm{SN}$ ) et un procès mis en saillance (SV), et (b) une hiérachie interlocutive avec (a) un terme de départ correspondant à un savoir mis en scène comme partagé (thématique, anaphorique, présupposant) et (b) un terme d'arrivée correspondant à un savoir possédé par le locuteur, mais supposément ignoré del'allocutaire. Sous cet angle, le français progresse du connu vers l'inconnu, à l'inverse de ce que fait le breton, et en porte-à-faux par rapport au basque, dont la logique est plutôt celle d'une synthèse (même si l'ordination des nominaux initiaux doit beaucoup aux faits de thématisation et topicalisation, aux rapports à l'interlocuteur et au contexte avant); et le français présente trois niveaux d'analyse : une orthosyntaxe nucléaire (le couple SN-SV), une parasyntaxe périphérique optionnelle (tous les excursus possibles en amont et par insertion), et une hypersyntaxe prosodique (le paragraphe oral de Morel et Danon-Boileau, qui se projette diversement sur la proposition ou une chaîne de propositions) ; apprendre à parler français suppose la maîtrise du schème orthosyntaxique de base dans ses fluctuations avec les addenda parasyntaxiques et son articulation hypersyntaxique, et la manière dont le locuteur régule la production du sens pour soi ou autrui se cristallise dans cette articulation.

Le français se caractérise également par l'omniprésence des éléments introducteurs (déterminant du nom, préposition du GN, sujet du SV, auxiliaire du verbe, pronom relatif et conjonction de la subordonnée), c'est-à-dire des opérateurs d'amorçage, indispensables même quand ils sont «sémantiquement vides » ou réputés tels, comme il impersonnel de il pleut. Prenons le cas de l'article. En basque, tout élément lexical est post-déterminé : etxea 'maisonla' « la maison »; dans le schéma lexis-nexus, le locuteur est entraîné à d'abord convoquer une catégorie notionnelle lexicale et ensuite la relier à une occurrence (par le déterminant) et à d'autres GN (par les postpositions comme l'ergatif). L'horloge mentale se résume à cette séquence notion-relations. En breton, le déterminatif est soit antéposé s'il est grammatical (déterminants, possessifs), soit postposé s'il est lexical (complément du nom déterminatif par sa position, sans préposition, comme l'annexion en arabe) : an ti «la maison », ti ma zad 'maison mon père' « la maison de mon père » ( $t i$ est post-déterminé lexicalement par ma zad, alors que zad est prédéterminé grammaticalement par $m a$ ). En gros, un déterminant grammatical et formel est thématisé, alors qu'un déterminatif lexical est rhématisé avec un effet heuristique dû à sa spécificité notionnelle, ce qui est «logique ». Un nom ne peut jamais être à la fois pré- et post-déterminé (*an ti ma zad) pour des raisons de redondance en chronosyntaxe ; en cas de récursivité, seule la dernière unité lexicale peut être prédéterminée grammaticalement, puisque toutes les autres sont post-déterminées lexicalement (ti amezeg skol-mestr ma zad 'maison voisin instituteur min père' " la maison du voisin de l'instituteur de mon père ». Il est clair que pré- et post-détermination sont deux manières différentes, l'une préconstruite et thématique (grammaticale) et l'autre improvisée et rhématique (lexicale), de réaliser la même opération, à savoir la corrélation de la classe notionnelle lexicale à une occurrence singularisée, référencielle ou non. Or en français, la prédétermination grammaticale n'est pas incompatible avec la post-détermination lexicale, et les deux ne sont pas redondants (la maison de mon père), signe que l'on n'a pas affaire à la même opération prise en charge à deux niveaux constructionnels distincts. En français, de même que le verbe doit toujours être amorcé par un sujet (même impersonnel), le nom doit toujours être amorcé par un article. D'un point de vue enactif et dans le domaine de la dynamique interprétative, cela signifie que l'interprétant est entraîné à toujours être prévenue de l'imminence d'une 
catégorie notionnelle-lexicale, laquelle est introduite par un marqueur de relation, de connexion classe-occurrence, qui spécifie par avance si la notion doit être reliée à une occurrence reconnaissable (article défini) ou une occurrence originale (article indéfini) : Je cherche un homme. Dans la plupart des cas, l'omission erronée de l'article ne rend pas nécessairement l'énoncé inintelligible, mais elle crée un effet de brutalité par lequel la notion a été présentée de manière non diplomatique, en court-circuitant le préliminaire : *Je cherche homme, *Passe-moi sel. En FLE, il est difficile de sensibiliser un russophone ou un nipponophone à l'effet cognitif de l'article, entraînés qu'ils sont à livrer directement la notion sans ce préliminaire relationnel essentiel dans l'interlocution. Pour y parvenir, on peut passer par des métaphores diplomatiques, telles que celle du facteur qui, livrant un paquet chez un résident, devrait obligatoirement presser un timbre grave pour déposer un courrier attendu (le défini) ou un timbre aigu pour annoncer un courrier imprévu (l'indéfini). Une fois la métaphore posée et clarifiée, on peut, en situation de cours, interrompre l'apprenant qui oublie l'article en protestant (« La sonnette !») et exigeant qu'il agisse intentionnellement sur l'allocutaire en sélectionnant l'article correspondant au timbre avertisseur désiré. Par cette démarche, l'apprenant se familiarise avec une approche consciente (donc déformée) de l'effet interactif de l'amorçage par l'article, et il lui reste ensuite à « déconscientiser » la routine et la transformer en ce que Janet nomme un automatisme. Cet apprentissage suppose une évolution de la manière de se relier à l'autre au moment de soumettre une unité lexicale, exactement comme le breton exige du francophone la découverte d'une manière de se relier à l'autre par le choix de la focale préverbale.

\section{Lexique}

Dans le cadre d'une approche enactive, le mot est envisagé comme une unité d'action, de nature motrice (le signifiant dans sa dimension articulatoire), couplée à des effets sensoriels habituels (le signifiant dans sa dimension perceptuelle multimodale : la perception du signal acoustique produit par soi-même ou autrui ; la perception visuelle de la motricité faciale d'autrui, en relation avec la proprioception tactile de sa propre motricité par le sujet parlant). Le caractère plus ou moins discret du mot comme unité d'action est une variable typologique : le mot des langues isolantes est constant, le mot flexionnel varie par la fin, le mot breton varie par le début (la mutation consonantique tad «père » / ma zad «mon père », le mot arabe varie par entrecroisement d'une matrice consonantique et d'un schème vocalique, le mot inuktitut agglutinant forme une holophrase - aussi la notion d'unité d'action est-elle à profiler au cas par cas.

Du point de vue enactif, prononcer un mot consiste à reproduire par l'action une expérience motrice et sensorielle analogue à des vécus antérieurs : chaque nouvel exemplaire est la reproduction d'une occurrence du signifiant analogue aux occurrences antérieures, dont le chaînage enregistré est nommé plexus analogique dans le modèle exemplariste du locuteur analogique de Lavie 2008. L'effet produit par ce geste lexical est la réactivation d'un réseau d'associations d'impressions, de connaissances et d'idées formé à l'occasion des expériences antérieures d'autres exemplaires, tous situés dans des situations pragmatiques vécues dont les interactions verbales elles-mêmes. Pour cette raison, la sémantique lexicale est à la fois extralinguistique et intralinguistique : pour un sujet donné, le mot chien active le réseau de traits collectés à l'occasion de l'ensemble des rencontres avec l'être-chien, directes et (l'animal), indirectes (dessins animés, films), avec médiation non verbale (images, photos, dessins) et verbale (tous les discours où le mot chien a été intégré, y compris : être chien avec quelqu'un, avoir du chien, réserver un chien de sa chienne, suivre quelqu'un comme un petit chien, s'entendre comme chien et chat). La production du mot est l'exécution d'une citation 
fragmentaire qui s'extrait d'un paradigme ouvert de discours antérieurs, ce qui lui confère la capacité de rouvrir un réseau de connaissances dialogiques, communautaires, qui concernent la manière habituelle dont s'utilise le mot dans la communauté et les propos habituels que l'on tient au moment où un exemplaire du mot est produit. Le réemploi du mot permet ainsi de se réapproprier, pour soi-même comme pour autrui, une tradition sémantique particulière.

Ce mécanisme est particulièrement important dans une langue comme l'anglais. Dans son histoire, l'anglais a engrangé des lexiques issus de traditions celtique, puis romaine, puis germaniques (Angles et Saxons), puis scandinaves (avec actuellement une controverse sur la primauté de la souche scandinave), de manière directe (les Vikings de la Danelaw au nord) et indirecte (les Vikings via le normand de Guillaume le Conquérant; et avant cela, les invasions Vikings en territoire germanique antérieures à l'invasion Anglo-Saxonne). Après cette série d'invasion s'ajoute le mouvement inverse, le contact linguistique lié aux multiples excursions anglaises hors des îles Britanniques (guerre de cent ans, Empire) et aux échanges culturels (Renaissance, Lumières, Révolution industrielle, révolution multimedia, et finalement la mondialisation sous toutes ses facettes).

Cet enchevêtrement a introduit dans le lexique anglais des mots issus de traditions diverses pour cristalliser des concepts réputés analogues, comme liberty et freedom pour « liberté » ou reach et attain pour "atteindre », et un observateur extérieur du système, par exemple un apprenant francophone, tend à les considérer comme interchangeables. Or, ce qui caractérise la valeur de ces paires oppositives et distinctives (au sens saussurien), c'est le fait que chacun, en tant qu'exemplaire singulier, s'extrait d'une lignée spécifique d'emplois traditionnels, laquelle peut être caractéristique du mode de vie et de pensée de la communauté langagière qui l'a introduit, par exemple les tribus germaniques du $6^{\mathrm{e}}$ siècle vs les intellectuels anglais du $19^{\mathrm{e}}$ siècle. Par exemple, reach, typiquement germanique, signifie le geste «atteindre » tel qu'on en fait l'expérience corporelle et matérielle dans la vie empirique et pragmatique : $\mathrm{He}$ reached for the bottle on the shelf « il étendit le bras pour saisir la bouteille sur l'étagère » (énoncé difficile à traduire en français : l'anglais du quotidien explicite, par habitude, les gestes précis dans leur dimension motrice et sensorielle plus que le fait le français, aussi une traduction trop sourcière semble-t-elle incongrue). Par contraste, attain signifie le geste conceptuel d'atteindre une cible abstraite par un mouvement de l'esprit : I doubt that we will attain this goal «je doute que nous atteignions cet objectif (discours formalisé, dans le cadre d'un exposé). Le même locuteur pourrait formuler reach this goal dans le cadre d'une interaction moins formelle, comme une discussion entre collègues se fréquentant habituellement. Les emplois de reach vs attain permettent au sujet parlant d'invoquer des traditions discursives et interactionnels antinomiques et de se mettre en scène, se produire (au sens théâtral) à l'instant de parole comme un acteur énonciatif identifiée à l'une ou l'autre lignée dialogique, et par là même se sculpter une identité momentanée conforme à des stéréotypes culturels divers, hérités d'apports communautaires multiples.

De la même manière, freedom (germanique, cf. allemand frei) signifie la liberté pragmatique d'agir sans entraves dans un environnement ouvert (cf. le titre du film Cry freedom ou le verbe free comme dans le slogan Free Nelson Mandela), alors que liberty invoque la notion philosophique de liberté de droit comme trait inhérent à la condition humaine (et dans sa dimension sociale, comme le début du Contrat Social de Rousseau) : liberty of speech (356000 occurrences google) est le terme juridique de référence alors que freedom of speech (55 millions d'occurrences la manière populaire d'en parler, raison pour laquelle on parle de limits to freedom of speech (87400 occurrences) et non pas de limits to liberty of speech (10 occurrences seulement, pratiquement des hapax, dont certains émanent de locuteurs identifiés, non anglophones). Plus révélateur encore, on distingue la célèbre Statue of Liberty (Manhattan), éloge philosophique de la liberté, de la Statue of Freedom (couple du Capitole à Washington), commencée par Crawford en 1860 pendant la Guerre de Sécession et porteuse 
de symboles anti-esclavagistes empruntés à la tradition romaine (le bonnet de l'esclave émancipé). Le verbe free signifie libérer d'entraves (prison, esclavage) alors que liberate signifie l'accès à un espace ouvert de liberté de choix et d'action. Très généralement, les choix lexicaux en anglais permettent au locuteur de distribuer des rôles culturels à l'interprétation aux partenaires impliqués (et aux interlocuteurs la capacité de les identifier) : « détruire » par accident ou vandalisme se dit destroy, mais «procéder à la destruction » d'un satellite en perdition se dit destruct ; «utiliser » un objet de la vie courante se dit use, mais «mettre en exploitation» des ressources naturelles jusqu'ici délaissées se dit utilize. Un francophone qui utilise attain, destruct et utilize à contre-emploi distribue sans le savoir des rôles non pertinents aux partenaires impliqués (même si ces mots passent pour synonymes et commutables dans de nombreux dictionnaires), rendant le message quelque peu incongru, avec le risque de mettre à mal sa propre image dans un milieu exigeant.

La compréhension de l'effet d'une unité lexicale donnée par aussi par l'identification de ses composantes submorphémiques le cas échéant. Les éléments submorphémiques sont entre autres des marqueurs à caractère phénoménologique, qui spécifie une classe d'expérience sensorimotrice saillante dans l'expérienciation ordinaire d'un processus ou d'un objet donné. Par exemple, la matrice sw- a été identifiés comme activant la simulation d'un balancement, mouvement oscillatoire ou pendulaire en anglais, et on la trouve dans des unités lexicales hétérogènes telles que swing «balancer», swim «nager», swoon «se pâmer», sway «osciller», swap «échanger» (mouvement par lequel on échange ses cartes professionnelles; vs trade et exchange). Il ne s'agit pas de phonosymbolisme, puisque la plupart des matrices inventoriées ne font guère état d'une quelconque connexion directe ou synesthésique à la perception auditive ou visuelle d'un phénomène. Diverses théories, dont la théorie sémio-génétique de Philps (2006, pour l'anglais) et le modèle matrices - étymons racines de Bohas (2010, pour les langues sémitiques) proposent des modèles élaborés de l'organisation de ces matrices en synchronie et leur développement diachronique dans l'optique d'une divergence d'activités biomécaniques non verbales et verbales. Pour notre propos, on retient simplement que la structure même du signifiant lexical peut jouer un rôle important dans la structure de la simulation mentale activée, et que cette congruence du signifiant et du signifié est en plein accord avec l'application du paradigme enactif au fait langagier. Ceci concerne également le domaine de la submorphologie grammaticale, avec parmi divers modèles la théorie des cognèmes, que l'on ne présentera pas ici, mais qui constitue l'un des volets de la linguistique enactive.

\section{Conclusion}

L'introduction d'une linguistique enactive et la «merleaupontysation » de l'objet linguistique apparaît comme la conséquence inévitable d'une réflexion phénoménologique renouvelée sur la nature du phénomène et le type de questionnement qu'il convient de lui appliquer, tant sur la nature du sens que sur le rôle de la corporéité interactive, socialisée, distribuée dans son avènement. L'intérêt de la démarche réside dans son aptitude à parler de l'expérience éprouvée par les sujets interagissant dans une langue donnée. Elle a quelque chose de révolutionnaire en ce qu'elle revendique le primat de l'action des corps sur les processus cognitifs, et elle peut en linguistique proposer une vision dynamique de la morphosyntaxe et de la sémantique lexicale qui tende à modéliser l'expérience des sujets et, de ce fait, expliciter des outils appropriés pour l'acquisition de langue seconde sous toutes ses formes. Dans le même temps, il est clair qu'une partie des affirmations de l'enactivisme hérite d'une tradition philosophique importante, et que la linguistique elle-même a réservé une certaine place à ces éléments, comme la psychomécanique du langage, l'appareil formel de l'énonciation de 
Benveniste, la théorie des opérations énonciatives de Culioli. Sous cet angle, la linguistique enactive explicite et assume la connexion philosophie / grammaire en revendiquant une construction phénoménologiquement motivée de ses outils descriptifs et analytiques et de ses objectifs, quitte à aboutir, selon les cas, soit à une reformulation améliorée de choses préexistantes, soit à de réelles innovations. L'importance accordée à l'expérience garantit que les modèles proposés aient une chance d'être parlants et, autant que possible, récupérables en vue d'applications diverses.

\section{Références}

ALEXAKIS V. 2010. Le premier mot. Stock.

ALEXAKIS V. 2002. Les mots étrangers. Stock.

ALEXAKIS V. 1995. La langue maternelle. Fayard.

BAKHTINE M. [1929] 1977. Marxisme et philosophie du langage. Paris : Minuit.

BERENDT J.-E. 1988. Die Welt ist Klang: Nada Brahma, Network-Medien-Cooperative, Frankfurt/M.

BERENDT J.-E. 1988. Vom Hören der Welt : Das Ohr ist der Weg, Network-MedienCooperative, Frankfurt/M.

Bergounioux G. 2004, Le moyen de parler, Paris : Verdier.

BERTHOZ A. 1997. Le sens du mouvement. Paris:Odile Jacob.

BoHAS G. 2006. The organization of the lexicon in Arabic and other semitic languages. In Perspectives on Arabic Linguistics XVI, Papers from the Sixteenth Annual Symposium on Arabic Linguistics, Cambridge, March, 2002, ed. S. Boudelaa, 1-37. Philadelphia: John Benjamins.

BotTineau D. 2010. «Language and enaction», J. Stewart, O. Gapenne, E. Di Paolo (eds), Enaction: toward a new paradigm for cognitive science, MIT, 267-306.

BotTineau D. 2011. « Le rôle de l'interculturalité dans l'enseignement de langues étrangères en école d'ingénieurs », Leeman D. (dir.), avec Cazade A., Chanlat J.-F., Louÿs G. et McEvoy S. (eds), L'interculturel en entreprise : quelles formations ? Limoges : Lambert Lucas, $115-126$.

BotTineau D. 2011. «Parole, corporéité, individu et société : l'embodiment entre le représentationnalisme et la cognition incarnée, distribuée, biosémiotique et enactive dans les linguistiques cognitives », Guignard J.-B. (dir), Linguistique cognitive : une exploration critique, Intellectica 56, 2011/2, 187-220.

BotTINEAU D. 2012. "Profondeur dialogique et morphosémantique lexicale et grammaticale », L. Begioni et C. Bracquenier (dir.). Sémantiques et lexicologie des langues d'Europe - Théories, méthodes, applications, Presses Universitaires de Rennes, 233-257.

BotTineau D. 2012. "La parole comme technique cognitive incarnée et sociale », Linguistique et phénoménologie du langage, La Tribune Internationale des Langues Vivantes, 52-53, Perros-Guirec : Anagrammes, 44-55.

BotTINEAU D. 2012. «Le langage représente-t-il ou transfigure-t-il le perçu ?», Formes sémantiques, langages et interprétations : Hommage à Pierre Cadiot, $\mathrm{n}^{\circ}$ spécial de La TILV (La Tribune Internationale des Langues Vivantes), F. Lautel-Ribstein (éd.), PerrosGuirec : Anagrammes, 73-82.

BotTineau D. 2012. «La fabrique de la langue, fabrique de l'humain », K. Nassikas, E. PrakDerrington, C. Rossi (eds), Fabriques de la langue, Presses Universitaires de France, 161197 
BotTinEAU D. 2012. «La poésie multilingue de Roy Eales : 'le corps entre quatre langues' ou la pratique de 'l'inverbation' », F. Lautel-Ribstein et J.-Y. Masson (éds.), Revue SEPTET, Des mots aux actes $n^{\circ} 3$ : Jean-René Ladmiral : une oeuvre en mouvement, Actes du colloque international organisé les 3-4 juin 2010 à l'université de Paris-Sorbonne, PerrosGuirec : Anagrammes, septembre 2012, 271-288.

ISBN : 978-2-84719-067-8

Bottineau D. 2012. «Submorphémique et corporéité cognitive», Miranda, n 7 (2012) Submorphemics / La submorphémique (Ed. Dennis Philps) - Celebrating Ceramics / Pleins feux sur la céramique (Ed. Helen Goethals) - December 2012, mis en ligne le 09/12/2012. URL :

http://www.miranda-

ejournal.eu/sdx2/miranda/article.xsp?numero=7\&id_article=Article_13-446.

BotTineaU D. 2012. «Syntaxe et interlocution en français et en breton », C. Douay \& D. Roulland (dir.). L'interlocution comme paramètre, Presses Universitaires de Rennes, 183206.

Bottineau D. \& Roulland D. 2007. «Le problème de la réflexivité en basque », A. Rousseau, D. Bottineau, D. Roulland (dir), L'énoncé réfléchi, Presses Universitaires de Rennes, 205-228.

CAdiot P. \& ViseTti Y.-M. 2001. Pour une théorie des formes en sémantiques, motifs, profils, thèmes, Paris : PUF.

Col G., Aptekman J., Girault S. \& Victorri B. 2010. «Compositionnalité gestaltiste et construction du sens par instructions dynamiques », Cognitextes 5, Numéro spécial AFLiCo 3 Grammaires en Construction(s), consulté le 01/04/2011 : http://cognitextes.revues.org/372

CotTE P. 1996. "Système de la syntaxe et refiguration du monde » in Cahiers de praxématique, 27, 129-48.

Culioli A. [1981] 1990. «Sur le concept de notion». BULAG $n^{\circ} 8$, repris dans Pour une linguistique de l'énonciation, t.1 : Opérations et représentations, Gap : Ophrys.

Clarke S. 2005. A Year in the Merde. London: Black Swan.

COWLEY S. 2009. «Distributed language and dynamics ». Pragmatics and Cognition 17(3), 495-507.

De JAEGHER H. \& Di PAOLO E.A. 2007. «Participatory sense-making : An enactive approach to social cognition ». Phenomenology and the Cognitive Sciences 6(4), 485-507.

DelatTRE P. 1953. Les modes phonétiques du français. The French Review, 27/1, 59-63.

DENEZ P. 1986. "Contraintes formelles de la structuration de la phrase bretonne », in CARRER, Philippe et alii (1986), Permanence de la langue bretonne, de la linguistique à la psychanalyse, Institut Culturel de Bretagne / Skol Uhel ar Vro, Rennes, 19-44.

DiNIS A. 2010. "From my-self to our-selves : persons as either 'individual substances' or 'relations' in Cowley Stephen J., Major João C., StefFensen Sune V., Dinis A. (Eds). 2010. Signifying Bodies: Biosemiosis, Interaction and Health, The Faculty of Portuguese Catholic University.Philosophy of Braga, 75-100.

DiXON R.M.W. 1994. Ergativity, Cambridge, C.U.P.

Dror I. E., HARNAD S. 2006. Cognition Distributed : how cognitive technology extends our minds. Amsterdam et Philadelphia : Benjamins.

EALES R. 1997. Lines on the Breton Edge. Pawel Pan Presse - Blackbird Editions.

EALES R. 2001. You can't change the way the rain appears... Blackbird Editions - Pawel Pan Presse. What matters most is what you make.

EALES R. 2004. Pawel Pan Presse - Blackbird Editions.

EALes R. 2008. On a Road to No End. Pawel Pan Presse - Blackbird Editions. 
EALES R. 2010. Just in case. Music / Poetry in five languages. CD indépendant. roy.eales@ orange.fr ou 02.96.35.92.61.

ERARD Y. 1998. "De l'énonciation à l'enaction. L'inscription corporelle de la langue », Cahiers de l'Institut de Linguistique et des Sciences du Langage, $n^{\circ} 11$, Mélanges offerts en hommage à Mortéza Mahmoudian, tome I et II.

FAUCONNIER, G. 1984. Espaces mentaux. Aspects de la construction du sens dans les langues naturelles. Paris : Editions de Minuit.

GIBSON J. J. 1977. The Theory of Affordances. In Perceiving, Acting, and Knowing, Eds. Robert Shaw and John Bransford.

GIBSON J. J. 1979. The Ecological Approach to Visual Perception

GOULD STEPHEN J., VRBA, E. 1982. "Exaptation - a missing term in the science of form", Paleobiology 8 (1982) : 4-15.

Guillaume G. 2003. Prolégomènes à la linguistique structurale 1. Québec: Les Presses de l'Université Laval.

Guillaume G. 2004. Prolégomènes à la linguistique structurale 2. Québec : Les Presses de l'Université Laval.

HAGEGE C. 1996. L'enfant aux deux langues. Odile Jacob.

JANET P. ([1889] 2005), L'automatisme psychologique - Essai de psychologie expérimentale sur les formes inférieures de l'activité humaine, L'Harmattan.

JANET P. 1926. De l'angoisse à l'extase. Paris. http://classiques.uqac.ca/classiques/janet_pierre/angoisse_extase_1/angoisse_1.html Chapitre I «l'ordre hiérarchique des tendances », 3. - les tendances moyennes.

JANET P. 1936. L'intelligence avant le langage. Paris. http://classiques.uqac.ca/classiques/janet_pierre/intelligence_langage/intelligence.html

JousSE M. 2008. L'anthropologie du geste. Paris : Gallimard, TEL.

KELLER P.-H. 2006. Le dialogue du corps et de l'esprit. Odile Jacob, Paris.

LADMIRAL J.-R. \& LIPIANSKY E.-M. 1989. La Communication interculturelle. Paris : Armand Colin.

LAFONT R. 1978. Le travail et la langue. Paris : Flammarion.

LAVELLE L. 1942/2005. La parole et l'écriture. Félin poche.

LAVIE R.-J. 2008. «Interspeaker variation and learnability in an exemplar-based productive model », in G. Desgulier, J.-B. Guinard \& J.-R. Lapaire (éds), Du fait grammatical au fait cognitif. From Gram to Mind (2 volumes), Bordeaux: Presses Universitaires de Bordeaux.

LEROI-GOURHAN A. (1964). Le geste et la parole. I. Technique et langage. II. La mémoire et les rythmes. Paris : Albin Michel.

MACCHI Y. 1986. «Du rôle du signifiant dans la genèse du sens énonciatif », Langages, 82, 67-82.

Liberman A.M. \& I.G. MATTINGLY. 1985. The motor theory of speech perception revised. Cognition 21: 1-36.

MAZAURIC C. 2005. "Fatigue d'être soi et mots étrangers: les Afriques de Vassilis Alexakis », Ethiopiques 74, http://ethiopiques.refer.sn/spip.php?page=imprimer-article\&id_article $=270$

MCFARLAND D. H. 2006. L'anatomie en orthophonie: parole, voix et déglutition. Atlas commenté. Masson.

MCGuRK, H., \& MCDONALD, J. (1976). "Hearing lips and seeing voices". Nature, 264, 23-30.

MENARY R. 2010. «Introduction to the special issue on 4E cognition ». Phenomenology and Cognitive Science 9 (2010) : 459-463.

MCNeILl D. Gesture and thought. Chicago: University of Chicago Press, 2005.

Merleau-Ponty, M. 1954. Phénoménologie de la perception. Paris : Gallimard. 
OGIEN A. 2007. Les formes sociales de la pensée, La sociologie après Wittgenstein. Paris : Colin.

Olivier G. La Cognition gestuelle. Ou de l'écho à l'égo. Presses universitaires de Grenoble.

PESCHARD I. 2004. La réalité sans représentation. La théorie enactive de la cognition et sa légitimité épistémologique. Doctorat de philosophie des sciences. Ecole Polytechnique.

PHILPS D. 2010. «Stratégies de nomination du corps et émergence du signe linguistique ». In

M. BANNIARD \& D. PhILPS (éds), La fabrique du signe, Linguistique de l'émergence, Toulouse : Presses Universitaires du Mirail, Interlangues, linguistique et didactique, 281298.

Rizzolatti G. \& Craighero L. 2004. "The mirror-neuron system”. Annual Review of Neuroscience, 27, 169-192.

SimONDON G. 1995. L'individu et sa genèse physico-biologique. Paris : Jérôme Millon.

STEWART J. (1996). Cognition = Life : Implications for higher-level cognition. Behavioural Processes 35: 311-326.

TOMATIS A. 1991. Nous sommes tous nés polyglottes. Fixot.

UEXKÜLL J. von [1934] 2004. Mondes animaux et monde humain. Pocket.

VALIN R. 1981. Perspectives psychomécaniques sur la syntaxe, Laval: Les Presses de l'université Laval, Québec.

Varela F., Thompson E. \& Rosch E. 1993. The Embodied Mind: Cognitive Science and Human Experience. MIT Press, Cambridge.

VYGOTSKY L.S. (1962). Thought and Language. E. KAUFMANN \& G. VAKAR, eds \& trans., Cambridge: MIT Press. 